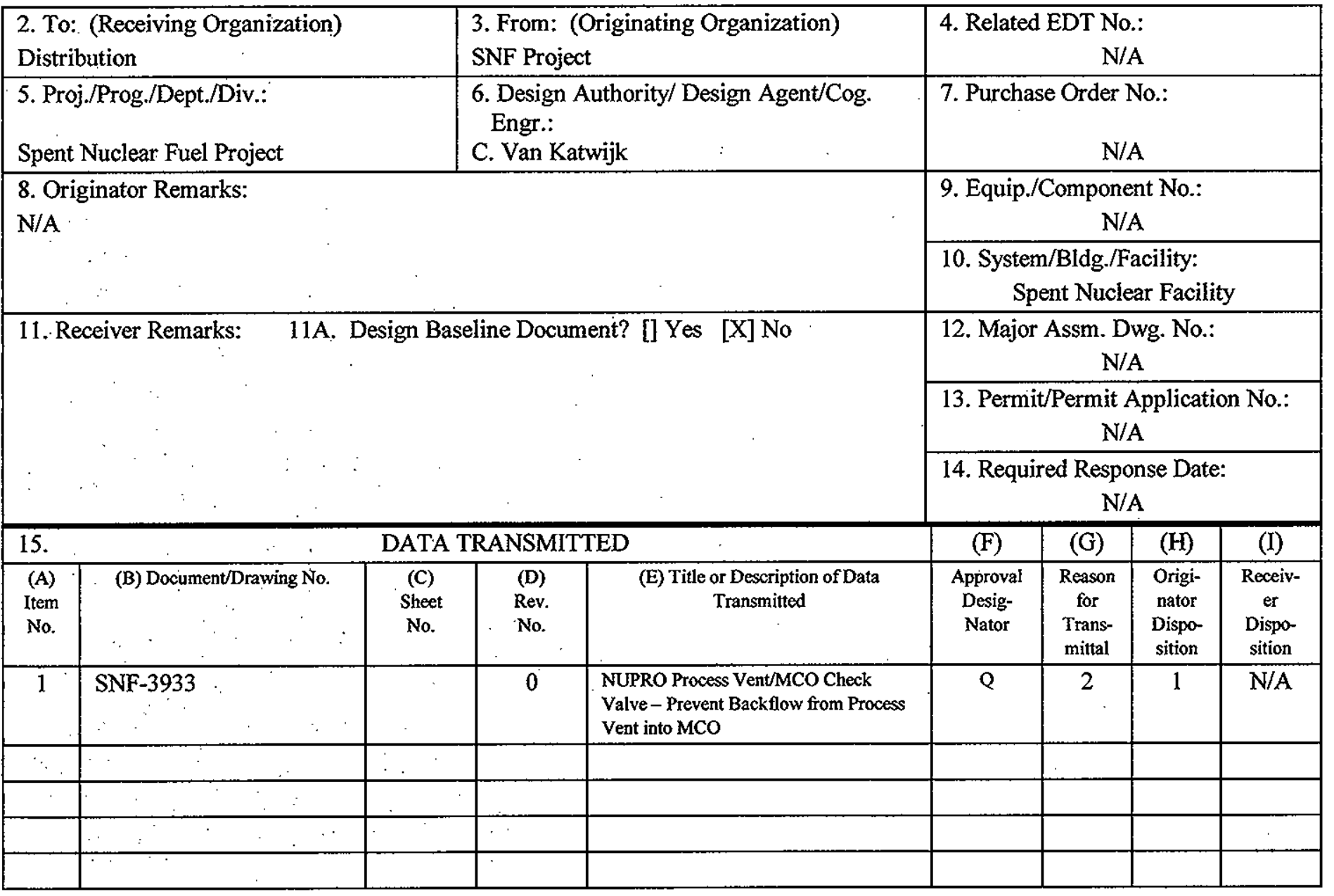

16.

\begin{tabular}{|l|}
\hline \multicolumn{1}{|c|}{ Approval Designator (F) } \\
\hline E, S, Q, D or N/A \\
(see WHC-CM-3-5, \\
Sec.12.7)
\end{tabular}

Sec.12.7)

\begin{tabular}{|ll}
\hline & Reason for Transmittal (G) \\
\hline 1. Approval & 4. Review \\
2. Release & 5. Post-Review \\
3. Information & 6. Dist. (Receipt Acknow. Required) \\
\hline
\end{tabular}
17. SIGNATURE/DISTRIBUTION

KEY

(See Approval Designator for required signatures)

\begin{tabular}{|c|c|c|c|c|c|c|}
\hline $\begin{array}{c}\text { (G) } \\
\text { Rea- } \\
\text { son }\end{array}$ & $\begin{array}{c}\text { Disp. } \\
2\end{array}$ & 1 & Designated Engineer C. Van Katwijk & $\begin{array}{c}\text { (G) } \\
\text { Rea- } \\
\text { Son }\end{array}$ & $\begin{array}{c}\text { (H) } \\
\text { Disp. }\end{array}$ & (J) Name (K) Signature (L) Date (M) MSIN \\
\hline 2 & 1 & Design Authority J. J. Irwin & (K) Signature (L) Date (M) MSIN & & & \\
\hline 2 & 1 & QA T. D. Hays & & & \\
\hline & & & & & \\
\hline & & & & & \\
\hline & & & & & \\
\hline
\end{tabular}
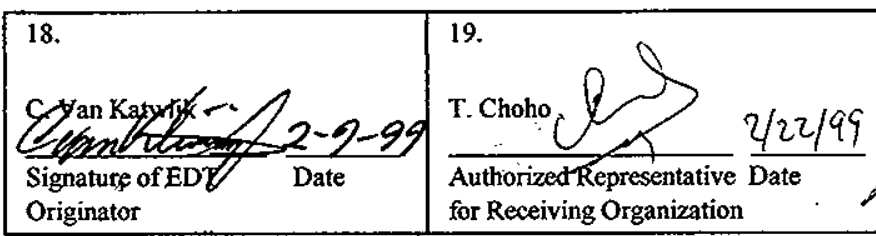

for Receiving Organization

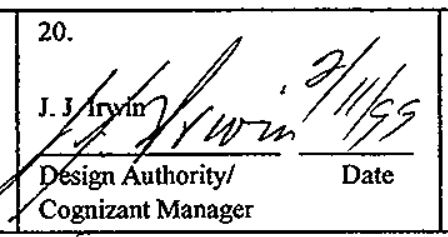

21. DOE APPROVAL (if required)

Ctrl. No.

[] Approved

D Approved w/comments

I Disapproved w/comments 


\title{
NUPRO Process Vent / MCO Check Valve - Prevent Backflow from Process Vent into MCO
}

\author{
Carl Van Katwijk \\ Numatec Hanford Co, Richland, WA 99352 \\ U.S. Department of Energy Contract DE-AC06-96RL13200 \\ EDT/ECN: 626282 \\ UC: 620 \\ Org Code: $2 \mathrm{G} 300$ \\ Charge Code: 105559/A000 \\ B\&R Code: $39 \mathrm{EW} 40400$ \\ Total Pages: 13
}

Key Words: Process Vent - MCO Pressure

Abstract: NUPRO Process Vent/MCO Check Valve - Prevent Backflow from Process Vent into MCO CGI-SNF-D-30-3-P4-040

TRADEMARK DISCLAIMER. Reference herein to any specific commercial product, process, or service by trade name, trademark, manufacturer, or otherwise, does not necessarily constitute or imply its endorsement, recommendation, or favoring by the United States Government or any agency thereof or its contractors or subcontractors.

Printed in the United States of America. To obtain copies of this document, contact: Document Control Services, P.O. Box 950, Mailstop H6-08, Richland WA 99352, Phone (509) 372-2420; Fax (509) 376-4989.
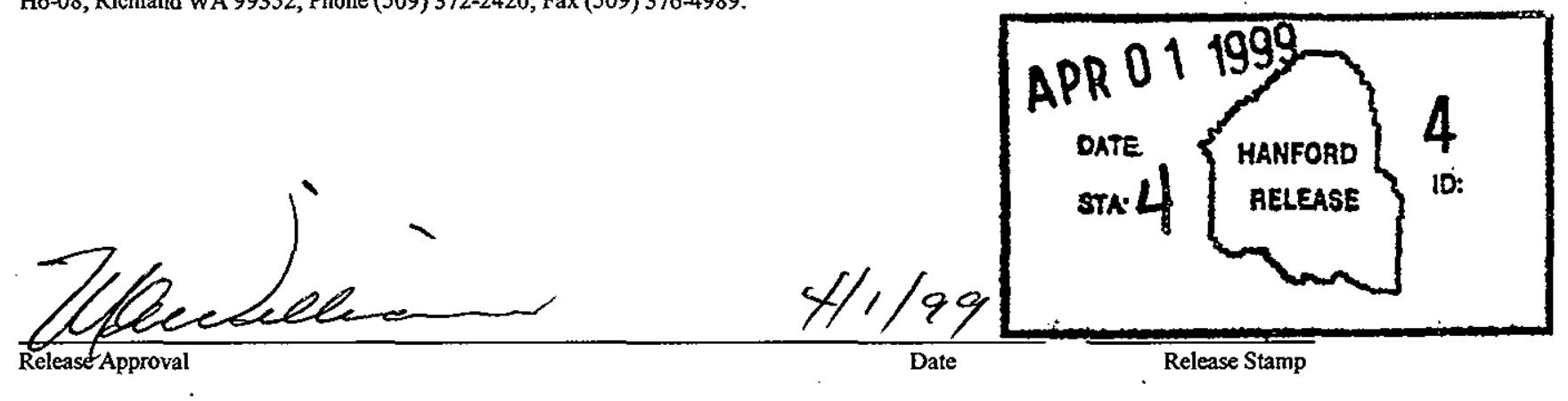
Commercial Grade Item Upgrade Dedication Form

ECN No. NA CGI No. CGI-SNF-D-30-3-P4-040

Rev. No. 0

Title: NUPRO PROCESS VENT/MCO CFECK VALVE - PREVENT BACK FLOW FROM PROCESS VENT INTO MCO

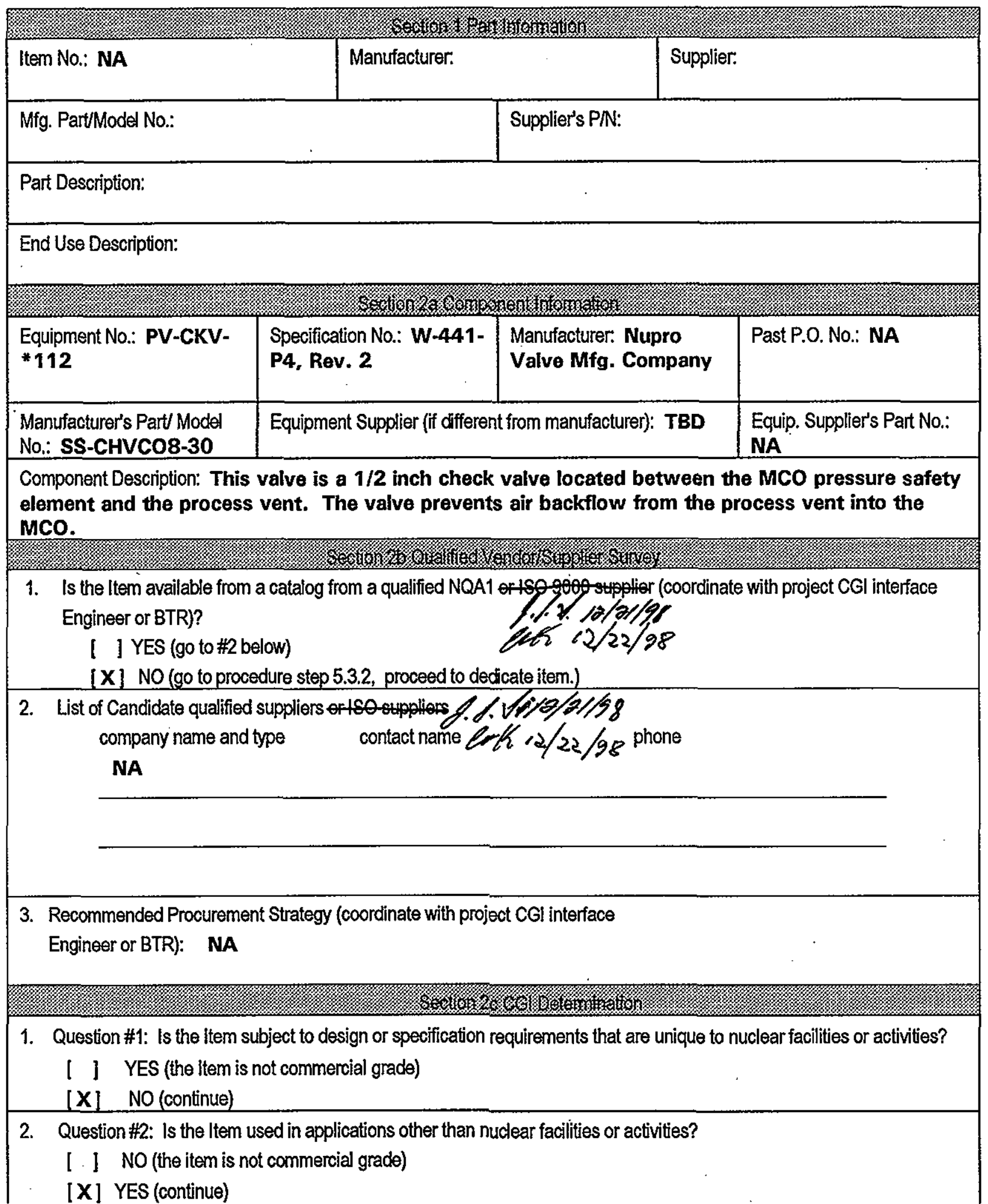


Commercial Grade Item Upgrade Dedication Form

ECNNo._NA

CGINo. CGI-SNF-D-30-3-P4-040

Title: NUPRO PROCESS VENT/MCO CHECK VALVE-PREVENT BACK FLOW FROM PROCESS VENT INTO MCO
Rev. No. 0

Page 2 of 12

SNG 3933

3. Question \#3: Is the ltem ordered from manufacturer/supplier on the basis or specifications set forth in the manufacturers catalog?

[ ] NO (the ltem is not commercial grade)

[X] YES (continue)

[X] All three criteria have been satisfied. The ltem meets the definition of commercial grade.

s

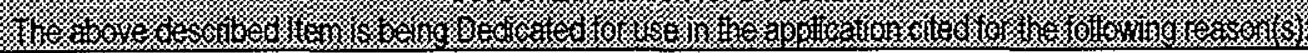

[X] Item is being purchased from a non ESL manufacturer supplier as commercial grade to be used in a Safety Class application.

[ ] Item is being purchased from a non ELS manufacturer supplier as commercial grade to be used in a Safety Significant application.

[ ] Item was purchased from a non ESL manufacturer supplier as commercial grade to be used in a Safety Class application.

[ ] Item was purchased from a non ESL manufacturer supplier as commercial grade to be used in a Safety Significant application.

[ ] Other ("like-for-like', similar, substitution, replacement evaluation)

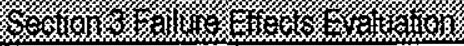

A. Part/Component Safety Function:

1. Check valve closure prevents air back leakage from the process vent to the MCO to prevent hydrogen explosion.

2. Confinement - Prevents air leakage into the MCO during vacuum operation or after rupture disc burst.

3. Maintain critical function during and after Seismic event.

B. Part/Component Functional Mode:

Safety Function \#1:

[X] Active-Mechanical or Electrical change of state is required to occur for the component to perform its safety function

[ ] Passive - Change of state is not required for the component to perform its safety function Safety Function \#2:

1 ] Active - Mechanical or Electrical change of state is required to occur for the component to perform its safety function.

[X] Passive - Change of state is not required for the component to perform its safety function Safety Function \#3:

[ ] Active-Mechanical or Electrical change of state is required to occur for the component to perform its safety function.

[X] Passive - Change of state is not required for the component to perform its safety function

C. Host Component Safety Function (if applicable):

1. NA

2.

3. 


\section{Error}

An error occurred while processing this page. See the system log for more details. 
Commercial Grade Item Upgrade Dedication Form ECNNo. NA CGINo. CGI-SNF-D-30-3-P4-040

Titie: NUPRO PROCESS VENT/MCO CHECK VALVE - PREVENT BACK FLOW FROM PROCESS VENT INTO MCO
Rev. No. 0

Page 4 of 12

SNe 3933

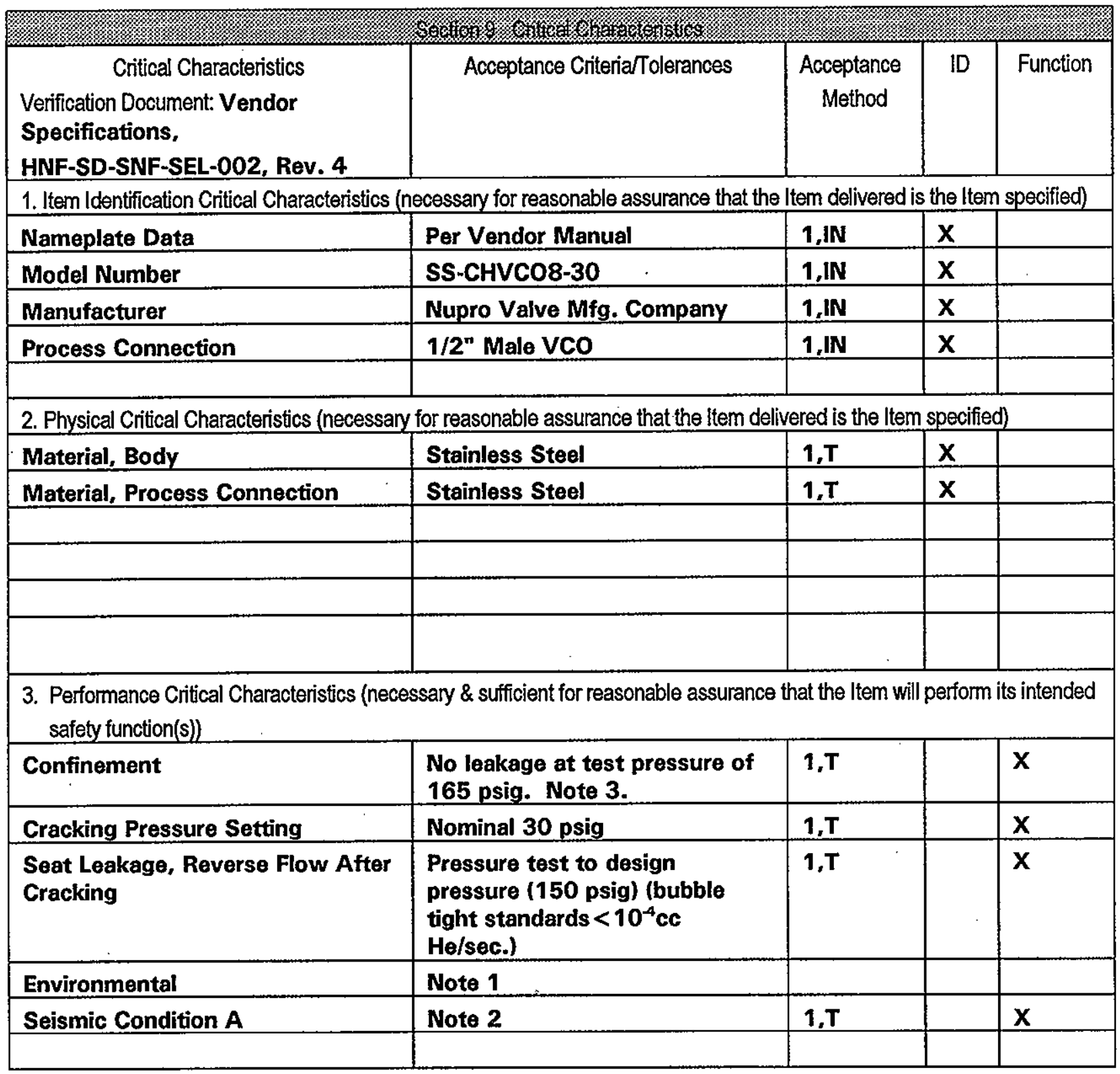




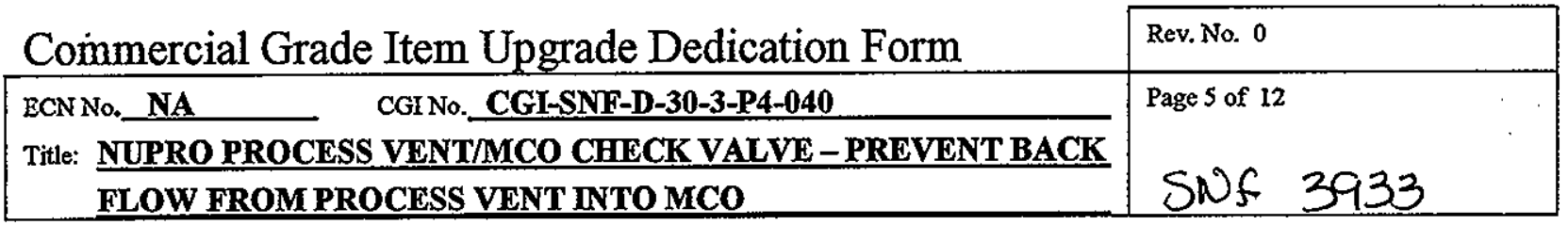

4. Notes and Legend:

1. The check valve packing (VITON) is not subject to degradation at ambient conditions of $40^{\circ} \mathrm{F}$ and $60 \% \mathrm{RH}$ or $115^{\circ} \mathrm{F}$ and $22 \%$ RH and is suitable for Environmental Condition B application.

2. Maintain critical function during and after Seismic event. $W$ 441-P4, Appendix L, page L-TBD provides a seismic testing plan for this safety class, Seismic Condition A component at a Seismic Spectra TBD.

3. Pressure test at $\mathbf{1 1 0 \%}$ of design accident condition pressure of 150 sig.
Acceptance Method:

1. Special Test and Inspection $1, \mathrm{IN}$ for Inspection 1,T for Test

2. Commercial Grade Survey

3. Source Verification

4. Vendor/ltem History

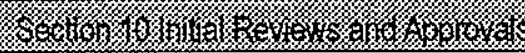

Approvals:

Designated Engineer: Cum Karen $12 / 22 / 98$

Design Authority:

QA Engineer

in $\frac{1}{\operatorname{man}} 12 / 22198$

$12 / 18 / 98$ 


\begin{tabular}{|c|c|c|}
\hline \multicolumn{3}{|c|}{$\begin{array}{c}\text { WORKSHEET } 1 \\
\text { DETERMINATION OF FALLURE MECHANISMSMODES }\end{array}$} \\
\hline \multicolumn{3}{|c|}{ 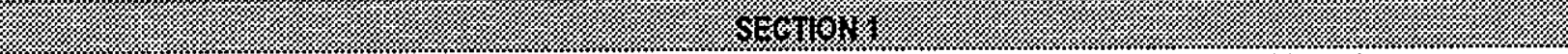 } \\
\hline $\begin{array}{l}\text { Typical Failure } \\
\text { Mechanisms }\end{array}$ & Definition & $\begin{array}{l}\text { Applicable to Component } \\
\text { under Evaluation }\end{array}$ \\
\hline Fracture & $\begin{array}{l}\text { Separation of a solid accompanied by little or no } \\
\text { macroscopic plastic deformation. }\end{array}$ & $\begin{array}{l}\text { Yes [] No [X]; if Yes, indicate failure } \\
\text { Mode. }\end{array}$ \\
\hline Comosion & $\begin{array}{l}\text { The gradual deterioration of a material due to } \\
\text { chemical or electrochemical reactions, such as }\end{array}$ & $\begin{array}{l}\text { Yes [ ] No } \quad \text { N ]; if Yes, indicate failure } \\
\text { Mode. }\end{array}$ \\
\hline Erosion & $\begin{array}{l}\text { Destruction of materials by the abrasive action of } \\
\text { moving fluids, usually accelerated by the presence }\end{array}$ & $\begin{array}{l}\text { Yes [] No [X]; if Yes, indicate failure } \\
\text { Mode. }\end{array}$ \\
\hline Open Circuit & $\begin{array}{l}\text { An electrical circuit that is unintentionally broken so } \\
\text { that there is no complete path for current flow. }\end{array}$ & $\begin{array}{l}\text { Yes [] No [X]; if Yes, indicate failure } \\
\text { Mode. }\end{array}$ \\
\hline Short Circuit & $\begin{array}{l}\text { An abnormal connection by which an electrical } \\
\text { current is connected to ground, or to some }\end{array}$ & $\begin{array}{l}\text { Yes [ ] No [X], if Yes, indicate failure } \\
\text { Mode. }\end{array}$ \\
\hline Blockage & $\begin{array}{l}\text { Clogging of a filtering medium resulfing in the } \\
\text { inability to perform its punification function or } \\
\text { blockace of flow. }\end{array}$ & $\begin{array}{l}\text { Yes [] No [X]; if Yes, indicate failure } \\
\text { Mode. }\end{array}$ \\
\hline Seizure & $\begin{array}{l}\text { Binding of a normally moving item through excessive } \\
\text { pressure, temperature, fiction, jamming. }\end{array}$ & $\begin{array}{l}\text { Yes [ ] No N ] ; If Yes, indicate failure } \\
\text { Mode. }\end{array}$ \\
\hline Unacceptable Vibration & $\begin{array}{l}\text { Mechanical oscillations produced are beyond the } \\
\text { defined permissible limits due to unbalancing, poor } \\
\text { supoort, or rotation at critical speeds. }\end{array}$ & $\begin{array}{l}\text { Yes [] No } \mathrm{X} \text { ]; if Yes, indicate failure } \\
\text { Mode. }\end{array}$ \\
\hline Loss of Properties & $\begin{array}{l}\text { A loss of mechanical and physical properties of a } \\
\text { material due to exposure to high temperatures, } \\
\text { radiation exposure. }\end{array}$ & $\begin{array}{l}\text { Yes [] No [X]; If Yes, indicate failure } \\
\text { Mode. }\end{array}$ \\
\hline Excess Strain & $\begin{array}{l}\text { Under the action of excessive external forces the } \\
\text { material of the part has been deformed or distorted. }\end{array}$ & $\begin{array}{l}\text { Yes [] No }[X] \text {; if Yes, indicate failure } \\
\text { Mode. }\end{array}$ \\
\hline Mechanical Creep & $\begin{array}{l}\text { From prolonged exposure to high temperature and } \\
\text { stress, the object will show a slow change in its } \\
\text { physical (shape and dimension) and mechanical }\end{array}$ & $\begin{array}{l}\text { Yes [ ] No NX]; if Yes, indicate failure } \\
\text { Mode. }\end{array}$ \\
\hline Ductile Fracture & $\begin{array}{l}\text { Fracture characterized by tearing of metal } \\
\text { accompanied by appreciable gross plastic } \\
\text { deformation. }\end{array}$ & No [X]; if Yes, indicate failure \\
\hline \multicolumn{3}{|c|}{ 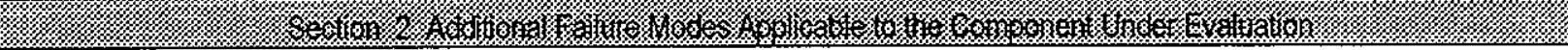 } \\
\hline \multicolumn{3}{|c|}{$\begin{array}{l}\text { 1. Failure of check valve to seat and prevent reverse flow/failure of } \\
\text { provide flow path for pressure safety element PV-PSE-1*33. }\end{array}$} \\
\hline \multicolumn{3}{|c|}{ 2. Process connection/body break. } \\
\hline
\end{tabular}




\section{CHECKLIST 1}

ACCEPTANCE METHOD 1

SPECIAL TEST/INSPECTION VERIFICATION

Item Description: Nupro Process Vent/Mco
Check Valve

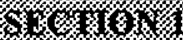

System \#: $30-3$

Manufacturer (Address/Phone):

Equip \#: PV-CKV-*112

Model \#: SS-CHVCO8-30

\begin{tabular}{|c|l|}
\hline Manufacturer (Address/Phone): & Supplier (Address/Phone):
\end{tabular}

Nupro Valve Mfg. Company

P.O. \#

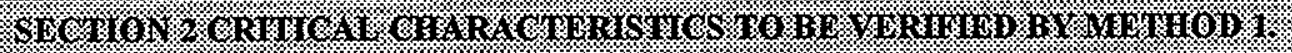

\begin{tabular}{|c|c|c|c|}
\hline Insp & Test & $\begin{array}{l}\text { Post } \\
\text { Test }\end{array}$ & \\
\hline$[\mathbf{X}]$ & [ ] & [ ] & 1. Nameplate Data \\
\hline$[\mathbf{X}]$ & [ ] & [ ] & 2. Model Number \\
\hline$[\mathbf{X}]$ & [ ] & [ ] & 3. Manufacturer \\
\hline$[\mathbf{X}]$ & [ ] & [ ] & 4. Process Connection \\
\hline [ ] & {$[\mathbf{X}]$} & [ ] & 5. Material, Body \\
\hline [ ] & {$[\mathbf{X}]$} & [ ] & 6. Material, Process Connection \\
\hline [ ] & {$[\mathbf{X}]$} & [ ] & 7. Confinement \\
\hline [ ] & {$[\mathbf{X}]$} & [ ] & 8. Cracking Pressure Setting \\
\hline [ ] & {$[\mathbf{X}]$} & [ ] & 9. Seat Leakage, Reverse Flow After Cracking \\
\hline [ ] & {$[\mathbf{X}]$} & [ ] & 10. Seismic Condition A \\
\hline
\end{tabular}

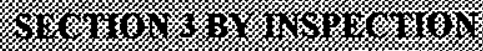

* See Attachment G of Desk Instruction for Sampling Size

Characteristic: Nameplate Data

Sample Size*: All Items

Acceptance Criteria: Per Vendor Manual

Receipt Inspection Plan / Report \#:

References (see Section 7): Nupro Poppet Check-Valves

Characteristic: Model Number

Sample Size*: All Items

Acceptance Criteria: SS-CHVC08-30

Receipt Inspection Plan / Report \#:

References (see Section 7): 


\begin{tabular}{|c|c|}
\hline Commercial Grade Item Upgrade Dedication Form & Rev. No. 0 \\
\hline ECN No. NA CGI No. CGI-SNF-D-30-3-P4-040 & Page 8 of 12 \\
\hline $\begin{array}{l}\text { Title: NUPRO PROCESS VENT/MCO CHECK VALVE-PREVENT BACK } \\
\text { FLOW FROM PROCESS VENT INTO MCO }\end{array}$ & sof 3933 \\
\hline
\end{tabular}

\section{Characteristic: Manufacturer \\ Sample Size*: All Items \\ Acceptance Criteria: Nupro Valve Mfg. Company \\ Receipt Inspection Plan / Report \#: \\ References (see Section 7):}

Characteristic: Process Connection

Sample Size*: All Items

Acceptance Criteria: 1/2" Male VCO

Receipt Inspection Plan / Report \#:

References (see Section 7):

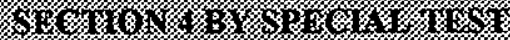

* See Attachment G of Desk Instruction for Sampling Size

\begin{tabular}{l|l} 
Test To Be Performed by: & Number of Items to be Tested:
\end{tabular}

[ ] Purchaser

[ ] Supplier/Manufacturer**

Test/Inspection Location:

[ ] Other

Characteristic for Test: Material, Body

Acceptance Criteria: Stainless Steel

Sample Size*: Normal Sampling Size

Actual Test Value:

Test Plan and Report\#: References (see Section 7):

Characteristic for Test: Material, Process Connection

Acceptance Criteria: Stainless Steel

Sample Size*: Normal Sampling Size

Actual Test Value:

Test Plan and Report \#: References (see Section 7):

Characteristic for Test: Confinement

Acceptance Criteria: No Leakage at Test Pressure of 165 psig

Sample Size*: Normal Sampling Size

Actual Test Value:

Test Plan and Report \#: References (see Section 7):

Characteristic for Test: Cracking Pressure Setting

Acceptance Criteria: Nominal 30 psig

Sample Size*: Normal Sampling Size

Actual Test Value:

Test Plan and Report \#: References (see Section 7): 
Commercial Grade Item Upgrade Dedication Form ECN No._NA $\quad$ CGINo._CGI-SNF-D-30-3-P4-040

Title: NUPRO PROCESS VENT/MCO CHECK VALVE - PREVENT BACK FLOW FROM PROCESS VENT INTO MCO

Characteristic for Test: Seat Leakage, Reverse Flow After Cracking

Acceptance Criteria: Pressure test to design pressure (150 psig) (bubble tight standards $<10-4 \mathrm{cc}$ $\mathrm{He} / \mathrm{sec}$.

Sample Size*: Normal Sampling Size

Actual Test Value:

Test Plan and Report \#: References (see Section 7):

Characteristic for Test: Seismic Condition A

Acceptance Criteria: Maintain Critical Function During and After Seismic Event

Sample Size*:

Actual Test Value:

Test Plan and Report \#: References (see Section 7):

**If Supplier/Manufacturer or Other, Refer to CGI Checklist-2 for Support Information 


\begin{tabular}{|c|c|c|c|c|c|c|c|c|c|c|c|}
\hline & & & & & & & & $\mathbf{X}$ & & 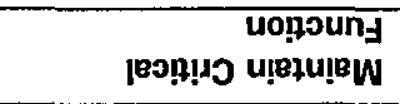 & $\forall$ uon!puog ग!us!̨s \\
\hline & & & & & & & & & & $\forall N$ & pequouudod!nug \\
\hline & & & & & & & & $\mathbf{X}$ & & 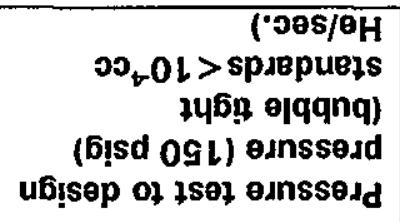 & 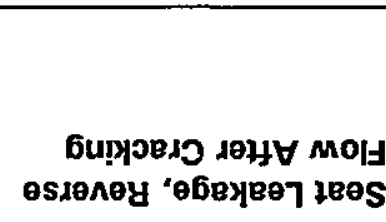 \\
\hline & & & & & & & & $\mathbf{x}$ & & 6!̣d oع jeuluoN & 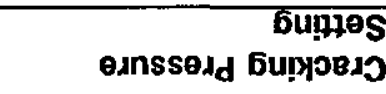 \\
\hline & & & & & & & & $\mathbf{X}$ & & 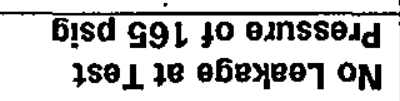 & fuøuəuyuog \\
\hline & & & & & & & & & $\mathbf{X}$ & jeөts sseju!gis & $\begin{array}{r}\text { uonjoutuos } \\
\text { ssejodd '[B!dejew }\end{array}$ \\
\hline & & & & & & & & & $\mathbf{X}$ & joets sseju!Ets & 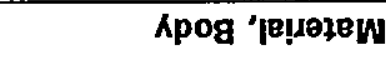 \\
\hline & & & & & & & & & $\mathbf{X}$ & 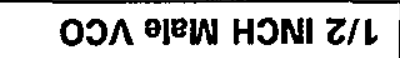 & uopoeuciog sseoodd \\
\hline . & & & & & & & & & $\mathbf{X}$ & 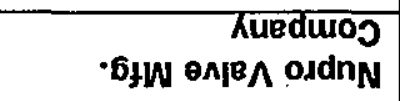 & dednłoejnuew \\
\hline & & & & & & & & & $\mathbf{x}$ & $0 \varepsilon-80 \supset \wedge H 0-S S$ & dequnN Iopow \\
\hline & & & & & & & & & $\mathbf{X}$ & |Bnuвw Lopuə $\wedge$ dod & elea elejdeueN \\
\hline भॄष्व & 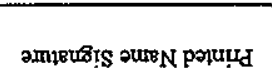 & 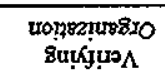 & 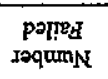 & $\begin{array}{l}\text { possol } \\
\text { roquank }\end{array}$ & 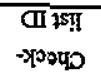 & $\begin{array}{l}\text { \#dA so } \\
\text { ampacosd }\end{array}$ & 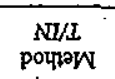 & norg̣poung & ஊ & 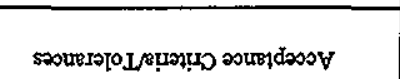 & 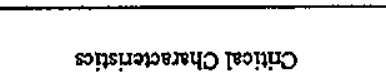 \\
\hline \multicolumn{8}{|c|}{ 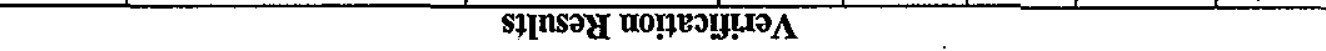 } & \multicolumn{4}{|c|}{ 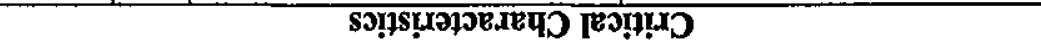 } \\
\hline & & & & & & & & & & & :NOILd\&OSGA WGJI \\
\hline \multicolumn{12}{|c|}{ 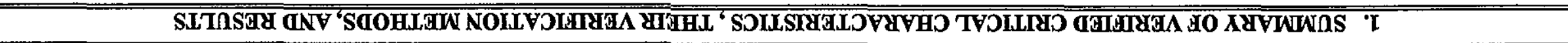 } \\
\hline & & & & 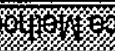 & (1) & Ty & (3) & 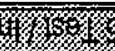 & (2) & & \\
\hline
\end{tabular}

\begin{tabular}{|c|c|}
\hline$\varepsilon \varepsilon b \varepsilon$ JNS & 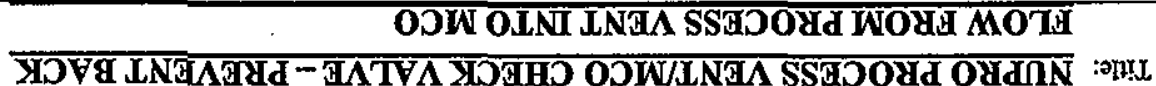 \\
\hline 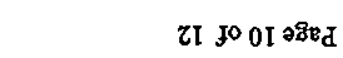 & 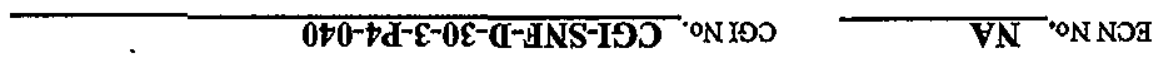 \\
\hline $0.0 N^{\circ} A P Y$ & นuı, \\
\hline
\end{tabular}


Commercial Grade Item Upgrade Dedication Form

ECN No._NA COXNo._CGI-SNF-D-30-3-P4-040

Rev. No. 0

Title: NUPRO PROCESS VENT/MCO CHECK VALVE - PREVENT BACK FLOW FROM PROCESS VENT INTO MCO

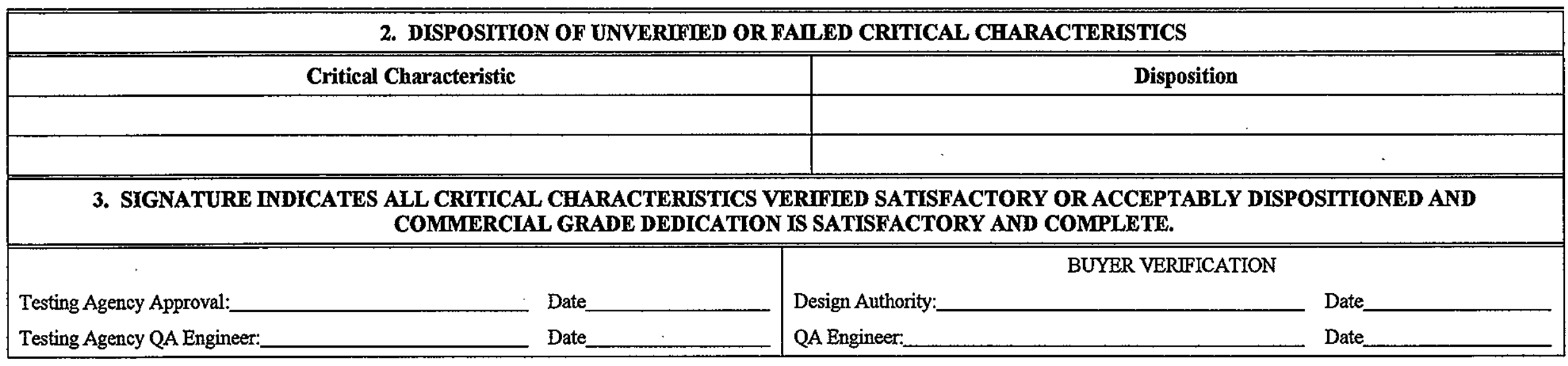




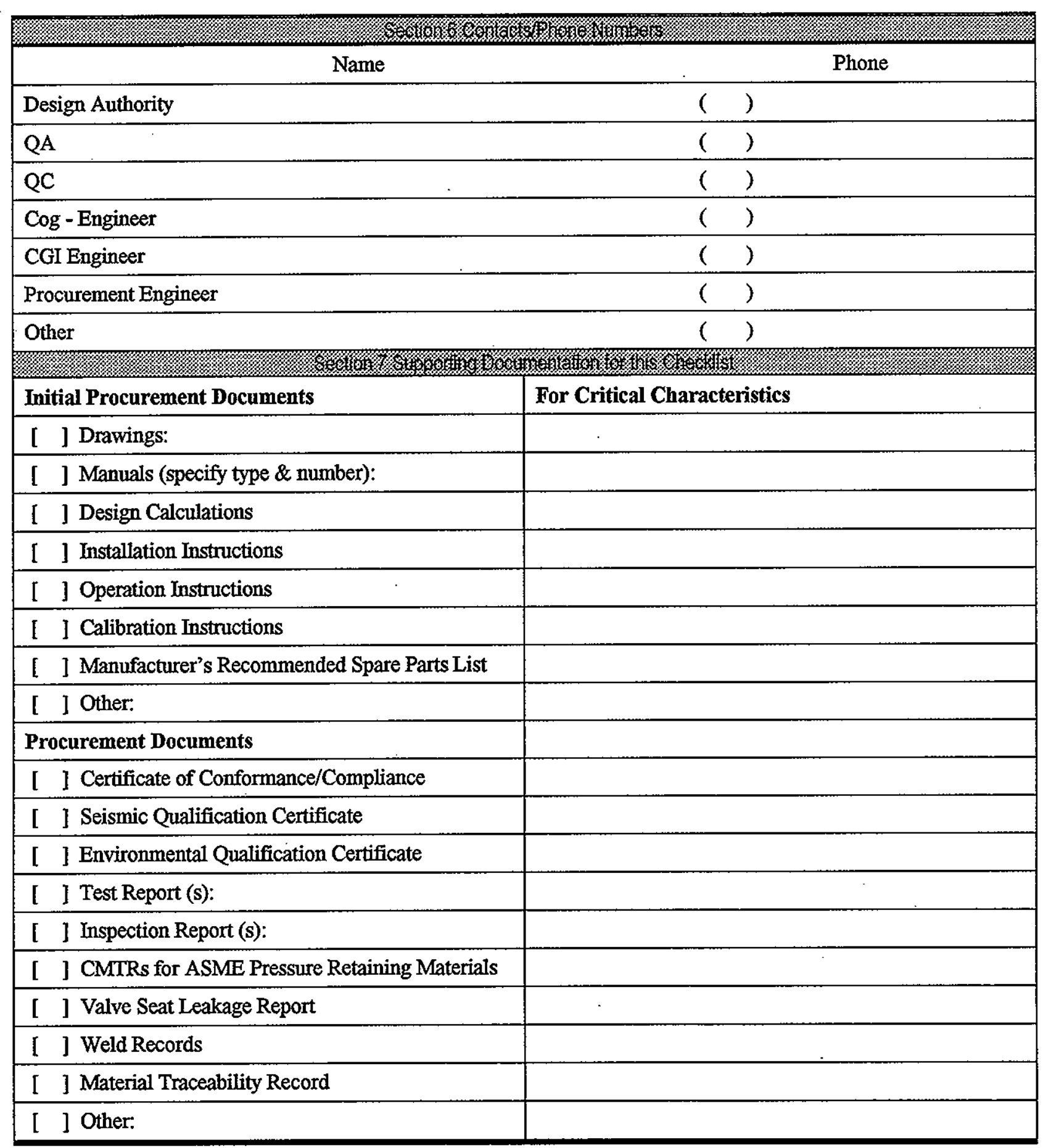

